\title{
ANSWER: Adaptive Network Selection in WLAN/UMTS EnviRonment*
}

\author{
Chih-Cheng Hsu ${ }^{1}$, Ming-Hung Chen ${ }^{1}$, Cheng-Fu Chou ${ }^{1}$, \\ Wei-Chieh Chi ${ }^{1}$, and Chung-Yi Lai ${ }^{2}$ \\ ${ }^{1}$ Dept. of Computer Science and Information Engineering, \\ National Taiwan University, Taipei, Taiwan, R.O.C. \\ \{Kenneth, mhchen, ccf, hypec\}@cmlab.csie.ntu.edu.tw \\ ${ }^{2}$ Institute for Information Industry, Taiwan, R.O.C. \\ laici@ii.org.tw
}

\begin{abstract}
The next generation wireless network is aimed to provide users with anywhere, anytime, and seamless service. With the increasing demand for the next generation network, many research works have focused on the efficient way to integrate different types of heterogeneous wireless networks such as cellular systems and wireless LAN. Therefore, the network selection technique plays a critical role in ensuring quality of service for the next generation network. In this paper, we propose an adaptive network selection (ANSWER) scheme, which is able to make the better decision about when to switch and choice on which access network. That is, we want to provide the always-bestconnected service as much as possible for the users. Specifically, to achieve the above goals, the available bandwidths of all possible networks, the location of user's device and its moving direction are taken into consideration in the ANSWER approach. We evaluate the performance of the ANSWER scheme through extensive simulations and the results agree with our goals.
\end{abstract}

Keywords: seamless, heterogeneous wireless network, available bandwidth, network selection.

\section{Introduction}

With the increasing demand for high data rate multimedia services, commercial thirdgeneration $(3 \mathrm{G})$ cellular network and handsets are gradually rolling into the market. Compared to cellular networks, WLAN (IEEE 802.11b offers a data rate up to $11 \mathrm{Mb} / \mathrm{s}$ ) is able to offer higher transmission bandwidth at a lower cost but cover smaller geographic areas. As a result, the WLAN is regarded as a proper candidate for high data rate services at certain hotspot areas with low user's mobility.

This work was partially supported by the National Science Council and the Ministry of Education of R.O.C. under the contract No. NSC95-2221-E-002-103-MY2 and NSC95-2622E-002-018. 
To provide anywhere, anytime, and seamless service, the next generation wireless network is expected to be a heterogeneous network, which can efficiently integrate several different characteristic access networks. Mobile users may move among these heterogeneous networks by seamlessly switching between different serving stations. How to take advantage of the wide coverage support of cellular network and the high data rates of WLANs is a challenge.

In cellular network, resource allocation is implemented by properly scheduling access to wireless channel to provide QoS guarantee. However, there is no user QoS guarantee in the current IEEE 802.11 WLAN standard. The latest IEEE 802.11e standard only enhances relative QoS. Besides, different traffic types usually require different QoS deliveries. Real-time services such as voice and video are sensitive to end-to-end delay, while the main concern of delay-tolerant data service is throughput. WLANs are more efficient than cellular networks in serving bursty data traffic, while it is quite difficult for WLANs to meet the strict delay requirements. Hence, the differences of QoS support in these two networks need to be considered for resource management.

While the moving speed of a mobile device is higher, more handoffs may occur during the lifetime of a call. The handoff procedure would cause extra delay, e.g., packet losses or even connection interruption. Moreover, handoff traffic should be separated from new traffic in terms of call admission. Thus, network selection algorithms and admission control for different requests are investigated.

In this work, we focus on finding the better access network between IEEE 802.11 WLAN and UMTS (Universal Mobile Telecommunications System) for the user device with two types of interfaces. Although the capacity of WLAN is larger than that of UMTS, the current available bandwidth of WLAN may decrease to be lower than the bandwidth guaranteed in UMTS when a lot of users stay in WLAN. In our proposed approach, we estimate the current available bandwidth of WLAN and the user dwell time in WLAN; then we make a handoff decision based on the information gathered. Since the coverage of UMTS network is much wider than that of WLAN, we assume that UMTS service always exists. Our contributions are estimating the network condition, predicting user's moving behavior and deciding if it is beneficial to make vertical handoff.

The remainder of the work is organized as follows. In section 2, we discuss related work about the UMTS-WLAN internetworking. In section 3, we describe the overall network selection algorithm including the basic idea, assumption, and detail procedures. In section 4, we evaluate the performance of proposed approach. Finally, we conclude the paper and give future works in section 5 .

\section{Related Work}

In traditional selection methods such as [1], only radio signal strength (RSS) threshold and hysteresis values are considered. However, they do not take the current condition or user's preference into account. When more handoff decision factors are considered, two-dimension cost functions, such as [2], are proposed. In one dimension, the function reflects the types of service $(\mathrm{ToS})$ requested by the user, and another dimension represents the cost to network according to specific parameters. The paper 
in [3] separates cost function factors into different categories: QoS factors, weighting factors, and network priority factors. QoS factors are defined based on user-specific requirements. The weight factors stand for the importance of the particular requirements with respect to the user. The network priority factors present the abilities of the networks to meet the requirements.

In [4], analytic hierarchy process (AHP) and the grey relational analysis (GRA) are integrated into the network selection algorithms. AHP is used to derive the weights of the parameters based on user's preference and service application. GRA takes charge of ranking the network alternatives. Some research works in [5] and [6] use dwelltimer to alleviate frequent handoffs and improve performance in transition area. They also investigate the effect of dwell-timer as the throughput ratio of WLAN and UMTS is changing. In [7], location information is shown to be beneficial to the accuracy of handoff decision in multi-service networks.

[8] [9] show that although the WLAN QoS capabilities have been extended with the introduction of IEEE 802.11e, the WLAN is still unable to support all QoS features provided by UMTS. Without affecting the service provided to existing WLAN data users, there is a limited number of UMTS roamers to be accepted in the WLAN depending on the bandwidth reservation and QoS requirements.

[10] presents a framework for a service provider to perform resource management in heterogeneous wireless networks. The proposed architecture allows the service provider to support real-time resource management functions based on Service Level Agreement (SLA) and seamless service handoff.

The experimental measurements of VoIP on 3G-WLAN internetworking system in [11] show that in addition to the VoIP connections, the performance of all clients in WLAN can degrade significantly due to the unfairness, undistinguished real-time and non real-time traffic of the packet queue in the AP and the inherent property of IEEE 802.11. In [12], they focus on the wireless multimedia distribution applications and recognize that service continuity is an important quality requirement. It designs and implements this class of applications on top of a session layer providing download continuity support when user changes location, network or terminal.

\section{ANSWER Framework}

In most of previous research works, the bandwidth in the WLAN is assumed to be greater than that in UMTS. However, for wireless networks, some conditions such as medium contention, channel fading, and interference, influence the available bandwidth. Furthermore, at the boundary of the WLAN cell, the received signal strength (RSS) varies around some thresholds. Such situation might cause frequently sequential vertical handoffs. This is not desired because the handoff procedure always demands extra time and no data traffic can be carried to or from mobile users during the procedure.

Therefore, instead of always switching to WLAN, evaluating the benefit of making vertical handoff is the main concern. The basic concept of our approach is: estimating the current available bandwidth of WLAN and predicting moving direction of mobile users to avoid unnecessary handoff. 


\section{Assumptions}

- $\quad$ Mobile IP allows users in an environment with two wireless networks.

- Mobile host is aware of its current position, speed, and direction by GPS.

- Coordinates of AP can be broadcast.

- UMTS network is always achievable and guarantee a certain amount of bandwidth.

Table 1. The definition of notations used in this work

\begin{tabular}{cc}
\hline Variable & Description \\
\hline$B W_{W L A N}$ & Available bandwidth in WLAN \\
$B W_{U M T S}$ & Available bandwidth in UMTS \\
$P_{r}$ & Receiving power of access point \\
$R X T r e s h$ & Receiving threshold in WLAN \\
$\triangle$ & Vertical handoff delay \\
$T$ & Sojourn time of the mobile host in WLAN cell \\
$v$ & Velocity of mobile host \\
$R$ & Transmission range of WLAN \\
\hline
\end{tabular}

Table 1 gives the definition of notations used in this work, $B W_{W L A N}$ is assessed by using the approach described in section 3.1. $B W_{U M T S}$ is a constant value since we assume that certain bandwidth is reserved for the mobile host. $P_{r}$ is measured from the packets sent from the base station. RXTresh is the minimal necessary power level for a packet to be successfully received. $\triangle$ comes from the vertical handoff procedure as the mobile host switches between different access networks. In section 3.2, we will introduce how we measure the value of $T$. $v$ is the current speed of the mobile host.

\subsection{Available Bandwidth Estimation}

The bandwidth estimation approach in [13] [14] is adopted in this article. Figure 1 shows the packet transmission sequence in IEEE 802.11. They measure the throughput $T P$ of transmitting a packet as $T P=S /\left(t_{r}-t_{S}\right)$, where $S$ is the size of the packet, $t_{s}$ is the time-stamp that the packet is ready at the MAC layer, and $t_{r}$ is the time-stamp that an ACK has been received. The time interval $t_{r}-t_{s}$ includes the channel busy and contention time. It is clear that the measured throughput of a packet depends on the size of the packet. A larger packet has higher measured throughput because it sends more data once it grabs the channel. To make the throughput

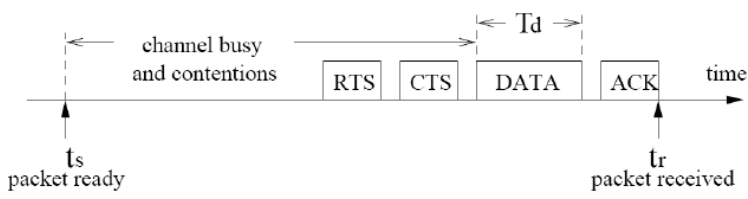

Fig. 1. IEEE 802.11 unicast packet transmission sequence 
measurement independent of the packet size, they normalize the throughput of a packet to a pre-defined packet size.

In Figure $1, T_{d}=S / B W_{c h}$ is the actual time for the channel to transmit the data packet, where $B W_{c h}$ is the channel's bit-rate. Here they assume channel's bit-rate is a pre-defined physical layer parameter. The transmission times of any two packets should differ only in their times to transmit the DATA packets. Therefore, the following equation holds:

$$
\begin{aligned}
& \left(t_{r 1}-t_{s 1}\right)-\frac{S_{1}}{B W_{c h}}=\left(t_{r 2}-t_{s 2}\right)-\frac{S_{2}}{B W_{c h}} \\
& \Rightarrow\left(t_{r 1}-t_{s 1}\right)-\frac{S_{1}}{B W_{c h}}=\frac{S_{2}}{T P_{2}}-\frac{S_{2}}{B W_{c h}}
\end{aligned}
$$

Where $S_{1}$ is the actual data packet size and $S_{2}$ is a pre-defined standard packet size. We can use Equation (2) to calculate normalized throughput $T P_{2}$ for the standard size packet and use the normalized throughput to represent the available bandwidth of wireless link.

\subsection{Sojourn Time Estimation}

We predict MH's sojourn time in WLAN by its current speed and its distance to the edge of WLAN cell. In the hotspot, MH simply moves with some velocity from one waypoint to another. As illustrated in Figure 2, the solid line represents the actual moving path of the $\mathrm{MH}$, and the dotted line stands for the path we predict the $\mathrm{MH}$ will move along. Since we know the moving direction of $\mathrm{MH}$ and the coordinates of both $\mathrm{MH}$ and AP, two vectors, $\vec{a}$ and $\vec{b}$, are obtained. By the definition of inner product:

$$
\vec{a} \cdot \vec{b}=|\vec{a}||\vec{b}| \cos \theta
$$

where $\theta$ is the angle between $\vec{a}$ and $\vec{b}, \cos \theta$ is derived. Then based on the cosine rule:

$$
R^{2}=|\vec{b}|^{2}+L^{2}-2 L|\vec{b}| \cos \theta
$$

The distance to the edge of WLAN cell, namely $L$, is obtained by equation (4). Therefore, the dwell time in WLAN is $T=L / v$, and even if $\mathrm{MH}$ is not in the AP coverage, $T$ represents the time to meet the boundary of WLAN.

\subsection{Network Selection Algorithm}

With the information gathered in 3.1 and 3.2, we describe the network selection procedure in Algorithm 1. Normally, the procedure should be executed for each probing interval $t$. However, in order to reduce overhead caused by frequently probing, we introduce a sleep time $t$ into the algorithm (Step 5 and 21). Step 1 to 5 gather the necessary information and stay in UMTS if no WLAN service is available. Once MH enters the AP coverage, the handoff decision is made based on the value cost. 


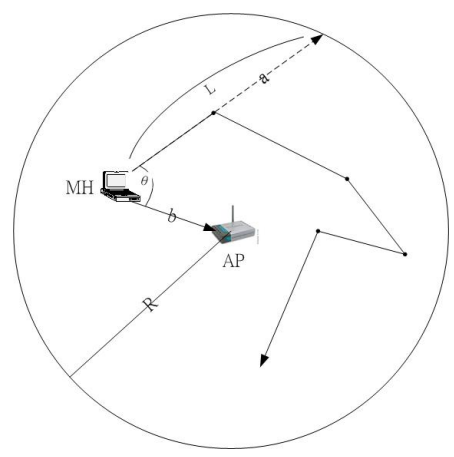

Fig. 2. Moving behavior of a mobile host

The idea of cost is: Is making handoff profitable for the throughput in next $t$ seconds? If MH is currently connected to UMTS and continues using UMTS service, no handoff is needed when MH leaves the WLAN. On the contrary, if MH switches to WLAN, two handoffs are made when MH switches back to UMTS. Similarly, if MH is currently connected to WLAN, only one handoff is made throughout the procedure no matter MH switches to UMTS or not. Thus, different situations result in different objective functions (Step 11 and 13). Note that we add $\alpha$ in the objective function to reduce oscillation. We prefer to stay in UMTS network when the bandwidth of WLAN approximates that of UMTS because WLAN network no longer has high bandwidth, which is its main advantage over UMTS network.

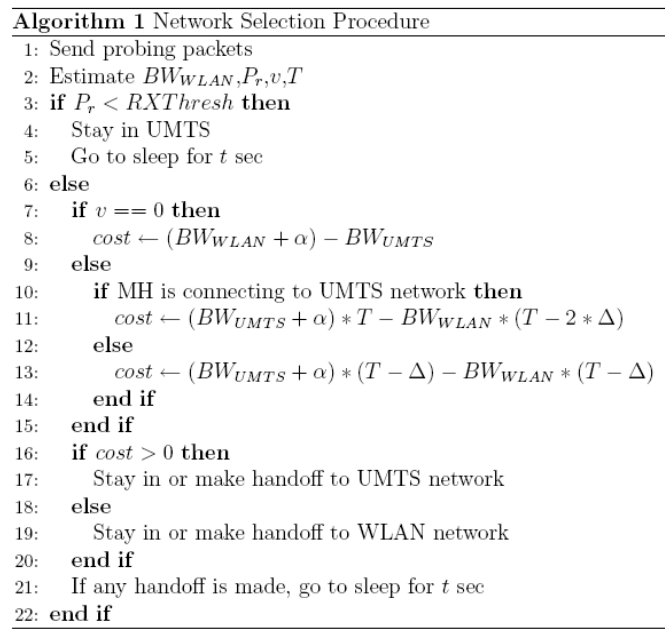

\section{Performance Evaluation}

We will show and discuss the performance of our approach compared with the WLAN-first and UMTS-first approach. Section 4.1 describes our simulation environment and performance metrics. 


\subsection{Network Environment and Performance Metrics}

To conduct our experiment, we use the NS-2 (Network Simulator 2) with the UMTS extension from [15]. We set up our WLAN-UMTS networks according to the parameters exposed in Table 2. Total simulation time is 500 seconds, and as to the data traffic destined to $\mathrm{MH}$, performances of CBR/UDP traffic are assessed respectively. Two metrics are used for our performance evaluation:

- Goodput: (total bits received - retransmitted bits) / measurement interval

- Number of handoffs: The quantity of handoffs MH made in its lifetime.

Table 2. Parameters used in performance evaluation

\begin{tabular}{cc}
\hline Parameter & Value \\
\hline Topology & $1000 \mathrm{~m} \times 1000 \mathrm{~m}$ \\
WLAN Capacity & $11 \mathrm{Mbps}$ \\
UMTS Capacity & $384 \mathrm{Kbps}$ \\
RXThresh & $3.6526 \mathrm{e}-10$ \\
Vertical handoff delay & $0.1 \mathrm{sec}$ \\
in WLAN & 10 to 60 \\
Number of Poisson background traffic & $1000 \mathrm{byte}$ \\
Packet size of Poisson traffic & $100 \mathrm{Kbps}$ \\
Average data rate of a Poisson traffic & $64 \mathrm{byte}$ \\
Probing packet size &
\end{tabular}

\subsection{Mobility Model}

Three most popular mobility models are used in our simulation, and the brief descriptions are the following:

- Random Waypoint Model: the Random Waypoint model is the most commonly used mobility model in research community. In the current NS-2, the implementation of this mobility model is as follows: at every instant, a node randomly chooses a destination and moves towards it with a velocity chosen uniformly randomly from $\left[0, V_{\max }\right]$, where $V_{\max }$ is the maximum allowable velocity for every mobile node. When reaching the destination, the node stops for duration. After this pause time, it again chooses a random destination and repeats the whole process again until the simulation ends.

- Freeway Mobility Model: this model emulates the motion behavior of mobile nodes on a freeway. Maps are used in this model. There are several freeways on the map and each freeway has lanes in both directions. The differences between Random Waypoint and Freeway are the following: (1) Each mobile node is restricted to its lane. (2) The velocity of mobile node is dependent on its previous velocity. (3) If two mobile nodes on the same freeway lane are within the safety distance, the velocity of the following node cannot exceed the velocity of preceding node. It also imposes strict geographic restrictions on the node movement by not allowing a node to change its lane. 
- Manhattan Mobility Model: Manhattan model emulates the movement pattern of mobile nodes on streets defined by maps. It can be useful in modeling movement in an urban area. The map of the Manhattan model is composed of a number of horizontal and vertical streets. The mobile node is allowed to move along the grid of horizontal and vertical streets on the map. At an intersection of a horizontal and a vertical street, the mobile node can turn left, right or go straight with certain probability. Except the above difference, the node relationships involved in the model are very similar to the Freeway model. However, it differs from the Freeway model in giving a node some freedom to change its direction.

The maximum speed and pause time in random waypoint model are $10 \mathrm{~m} / \mathrm{s}$ and 10 seconds respectively in our simulation. The speed in Freeway mobility model ranges from 10 to $60 \mathrm{~m} / \mathrm{s}$, and the maximum speed in Manhattan model is $10 \mathrm{~m} / \mathrm{s}$.

\subsection{Goodput Comparison}

From Figure 3 (a) to (c), we can see that our approach ANSWER greatly outperforms WLAN-first and UMTS-first method in CBR traffic, no matter network load in WLAN is heavy or not. Our approach will mostly stay in UMTS network when the WLAN is too crowded (number of background traffic is 30 to 50) and efficiently utilize the high bandwidth of WLAN when network load of WLAN is light (number

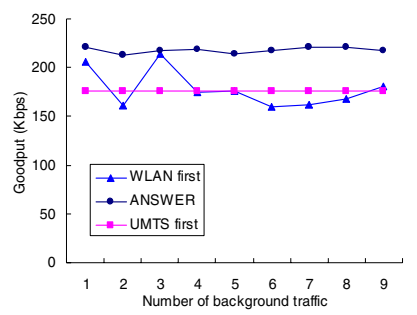

(a) Random Waypoint model (Number of background traffic: $1 \sim 9$ )



(c) Manhattan model (Number of background traffic: $10 \sim 50$ )

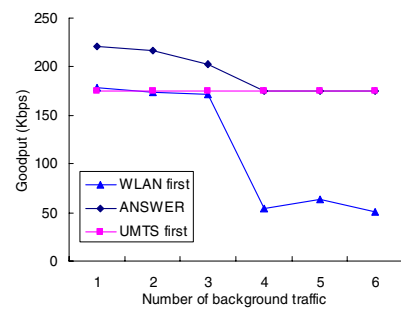

(b) Random Waypoint model (Number of background traffic: $10 \sim 50$ )

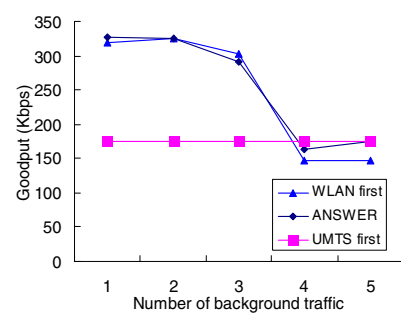

(d) Freeway model (Number of background traffic: $10 \sim 50$ )

Fig. 3. CBR goodput comparison in different mobility model 
of background traffic is 10 to 30$)$. In Figure 3(d), the performance of freeway mobility model is not good as that in the other two mobility model when the background traffic in WLAN increases, because MH rushes in and out as its moving speed is fast.

\subsection{Number of Vertical Handoffs}

Vertical handoff causes call intermission, so in this section we focus on how many handoffs $\mathrm{MH}$ makes throughout the whole course. Figure 4 show that number of handoff in our approach is much less than that in WLAN-first method. Our selection algorithm efficiently reduces many unnecessary handoffs by predicting MH's sojourn time.

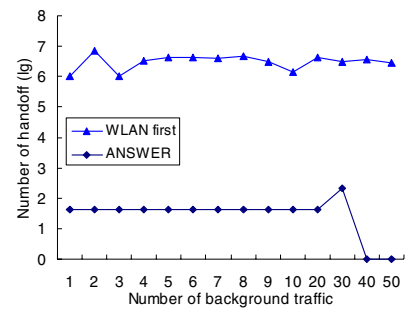

(a) Number of handoff in Random Waypoint model



(b) Number of handoff in Manhattan model



(c) Number of handoff in Freeway model

Fig. 4. Number of handoff in different mobility model

\subsection{Accuracy of Available Bandwidth}

To measure the accuracy of the estimated available bandwidth, we first assume the total usable bandwidth in WLAN to be the maximum WLAN utilization achieved in our simulations, which is about 3Mbps. Thus, the bandwidth unused by the Poisson background traffic is the approximation of actual available bandwidth. The comparison of the approximation and the estimated value by the approach in section 3.1 is in Figure 5.

When the network load is light, the bandwidth estimated is about $1700 \mathrm{Kbps}$ at most. The reason is that $t_{r}-t_{s}$ in Figure 5(a) has a minimum value since the 




(a) Comparison when Number of background traffic: 1 to 20

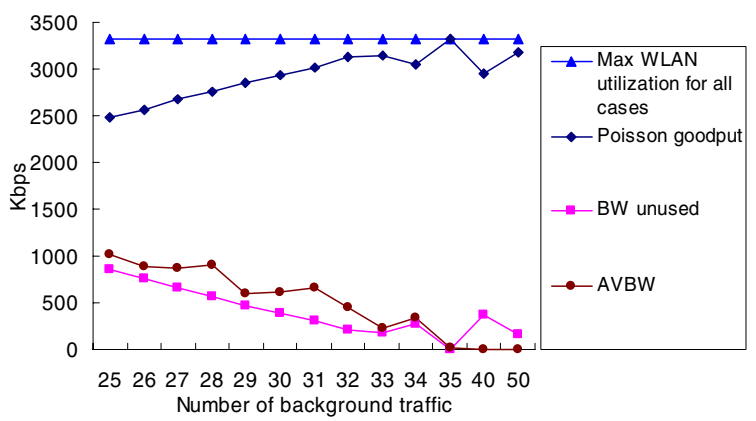

(b) Comparison when Number of background traffic: 21 to 50

Fig. 5. Comparison between estimated and actual bandwidth

contention period in IEEE 802.11 can not be reduced unlimitedly. When the load increases, the two curves become closer to each other. Although the estimated value is not highly precise, the two curves go in the same trend.

\subsection{Dynamic Sleep Time}

We adjust the sleep time $t$ in our algorithm based on speed and available bandwidth on the instant, as equation (5) shows.

$$
t=c \times \frac{B W_{W L A N}}{B W_{U M T S}} \times \frac{1}{v}
$$

CBR traffic destined to $\mathrm{MH}$ in Random Waypoint model is simulated and the number of background traffic is set to 30 . We can see that there is a tradeoff between goodput/jitter and number of handoff/probe in Figure 6. As $c$ increases, MH would miss some opportunities to switch to the better network in sleep time but it would send fewer probing packets. 


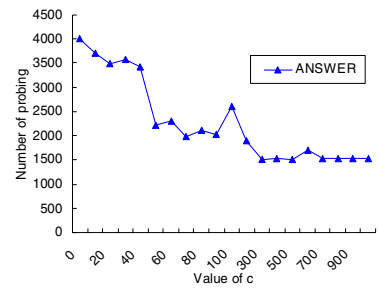

(a) Number of probe



(c) Goodput

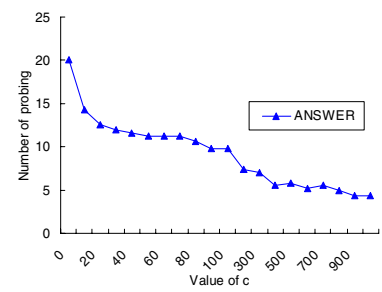

(b) Number of handoff

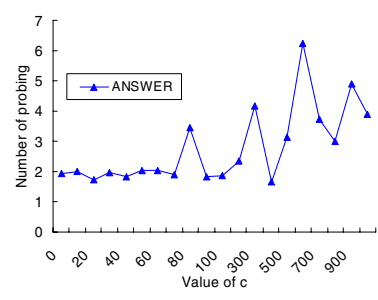

(d) Average jitter

Fig. 6. Simulation results for different value of $\mathrm{c}$

\section{Conclusion and Future Works}

In this work, we have proposed an approach dealing with network selection from WLAN and UMTS network. The approach estimates current available bandwidth and sojourn time in WLAN to choose the better network. The simulation in this paper shows that our algorithm achieves better performance and has fewer handoffs than UMTS/WLAN-first methods do in different mobility models. In addition, we investigate the accuracy of available bandwidth estimation, and the tradeoff between goodput and number of handoff.

It is noted that only two networks, WLAN and UMTS, are considered in our scheme. More types of network, such as WiMAX and WCDMA, will be integrated in our scheme. Moreover, we would like to adjust our approach for different traffic with different QoS.

\section{References}

1. Tripathi, N.D., Reed, J.H., Van Landingham, H.F.: Adaptive handoff algorithms for cellular overlay systems using fuzzy logic. In: Vehicular Technology Conference, 49th, vol. 2, pp. 1413-1418. IEEE, Los Alamitos (1999)

2. Park, H., Yoon, S.H., Kim, T.H., Park, J.S., Do, M.S., Lee, J.Y.: Vertical Handoff Procedure and Algorithm between IEEE 802.11 WLAN and CDMA Cellular Network. In: Mobile Commun. 7th CDMA Intl. Conf. 
3. Roveri, A., Chiasserini, C.F., Femminella, M., Melodia, T., Morabito, G., Rossi, M., Tinnirello, I.: The RAMON Module: Architecture Framework and Performance Results. In: Proceedings of the Second International Workshop on Quality of Service in Multiservice IP Networks (2003)

4. Song, Q., Jamalipour, A.: Network selection in an integrated wireless LAN and UMTS environment using mathematical modeling and computing techniques. Wireless Communications 12(3), 42-48 (2005)

5. Ylianttila, M., Pande, M., Makela, J., Mahonen, P.: Optimization scheme for mobile users performing vertical handoffsbetween IEEE 802.11 and GPRS/EDGE networks. In: GLOBECOM 2001. Global Telecommunications Conference, vol. 6, IEEE, Los Alamitos (2001)

6. Ylianttila, M., MÄakelÄa, J., Pahlavan, K.: Analysis of handoff in a location-aware vertical multi-access network. Computer Networks (2005)

7. Makela, J., Ylianttila, M., Pahlavan, K.: Handoff decision in multi-service networks. Personal, Indoor and Mobile Radio Communications. In: PIMRC 2000. The 11th IEEE International Symposium (2000)

8. Salkintzis, A., Skyrianoglou, D., Passas, N.: Seamless multimedia QoS across UMTS and WLANs. In: Vehicular Technology Conference, VTC 2005-Spring. 2005, 61th, vol. 4, IEEE, Los Alamitos (2005)

9. Salkintzis, A.K., Dimitriadis, G., Skyrianoglou, D., Passas, N., Pavlidou, N.: Seamless continuity of real-time video across umts and wlan networks: challenges and performance evaluation. In: Wireless Communications, vol. 12(3), pp. 8-18. IEEE, Los Alamitos (2005)

10. Yang, X., Bigham, J., Cuthbert, L.: Resource management for service providers in heterogeneous wireless networks. In: Wireless Communications and Networking Conference, vol. 3, IEEE, Los Alamitos (2005)

11. Rajavelsamy, R., Jeedigunta, V., Holur, B., Choudhary, M., Song, O.: Performance evaluation of VoIP over 3G-WLAN interworking system. In: Wireless Communications and Networking Conference, vol. 4, IEEE, Los Alamitos (2005)

12. Ghini, V., Salomoni, P., Pau, G.: Always-best-served music distribution for nomadic users over heterogeneous networks. Communications Magazine 43(5), 69-74 (2005)

13. Shah, S.H.H., Chen, K.H., Nahrstedt, K.H.: Dynamic Bandwidth Management in SingleHop Ad Hoc Wireless Networks. Mobile Networks and Applications 10(1), 199-217 (2005)

14. Shah, S.H., Chen, K., Nahrstedt, K.: Available Bandwidth Estimation in IEEE 802.11based Wireless Networks. In: Proceedings of 1st ISMA/CAIDA Workshop on Bandwidth Estimation (BEst)

15. EURANE. Enhanced UMTS Radio Access Network Extensions for NS-2, http://www.ti-wmc.nl/eurane/ 